\title{
ANALYSIS OF LIMIT CYCLE STABILITY IN A TAP-CHANGING TRANSFORMER
}

\author{
V. Donde \\ I.A. Hiskens \\ Department of Electrical and Computer Engineering \\ University of Illinois at Urbana-Champaign \\ 1406 West Green Street \\ Urbana, IL, 61801, USA
}

\begin{abstract}
The paper analyses the nature of limit cycles induced through the interaction of transformer tap changing and load dynamics. Linearization of a Poincaré map is used to prove local stability. An approximation is introduced which allows the map to be formulated explicitly. An estimate of the region of attraction can then be obtained.
\end{abstract}

\section{INTRODUCTION}

Interactions between tap-changing transformers and dynamic loads can result in limit cycle (periodic) behavior [1]. Analysis of this phenomenon is complicated by the discrete nature of transformer taps. In fact, this form of interaction, between discrete and continuous states, typifies hybrid systems. This paper takes a hybrid systems approach to analysing stability of the induced limit cycles.

\section{TAP CHANGER LOGIC}

Figure 1 shows a flowchart of the logic that characterizes an automatic voltage regulator (AVR) of a tap changing transformer. This device undergoes discrete changes in tap ratio when certain conditions are satisfied. The AVR is driven by a number of interacting events that govern timer behavior. The primary input is the voltage $V_{m}$ at a bus on the secondary side of the transformer. If $V_{m}$ lies within the deadband, i.e., $V_{L}<V_{m}<V_{H}$ or the tap is at the upper or lower limit, the timer is blocked. When the voltage deviates outside the deadband, the timer runs. If the timer reaches $T$, a tap change occurs. Tap increases by a single step if $V_{m}<V_{m}^{0}$, and decreases by a step if $V_{m}>V_{m}^{0}$. Once a tap change takes place, the timer is reset.

\section{SIMPLE POWER SYSTEM}

The example power system of Figure 2 shall be used to explore limit cycles induced by transformer tap changing. The system supplies a dynamic reactive power load via a transformer with turns ratio $1: n$. The dynamic load [2] is described by the differentialalgebraic model

$$
\begin{aligned}
\dot{x}_{q} & =\frac{1}{T_{q}}\left(Q_{s}-Q_{d}\right) \\
Q_{d} & =x_{q}+Q_{s} V^{2}
\end{aligned}
$$

where $Q_{s}$ and $Q_{d}$ are steady state and dynamic reactive powers respectively, with $Q_{s}$ constant, $x_{q}$ is the load state variable, and $V$
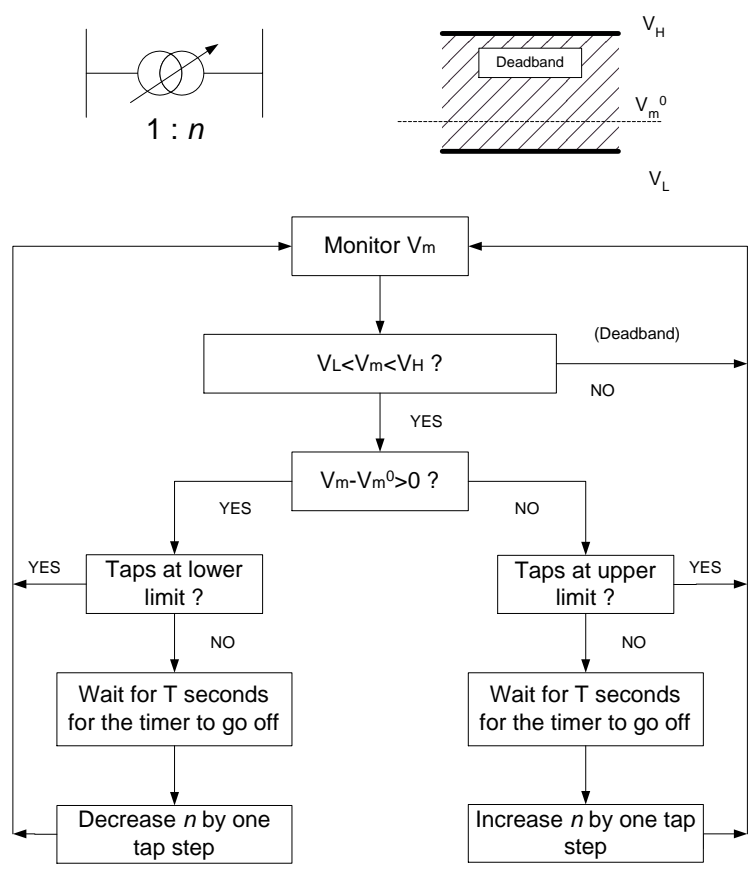

Fig. 1. Tap changer logic flowchart.

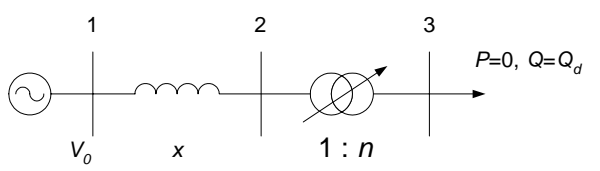

Fig. 2. Example system.

is the voltage at load bus 3 . The load responds dynamically with time constant $T_{q}$. Power balance at the load bus is given by

$$
Q_{d}=\frac{V\left(n V_{0}-V\right)}{n^{2} x}
$$

where $n \in \mathbf{Q}$ is the (discrete) tap position, and $\mathbf{Q}$ is the countable set of discrete tap positions.

As the tap changing logic drives the system towards $V=$ $V_{m}^{0}=1$, (stable and/or unstable) equilibria will exist in neighborhoods of the intersections of the $V=1$ and $\dot{x}_{q}=0$ curves in $x_{q}-n$ space $[3,4]$. For the example system, these curves are 


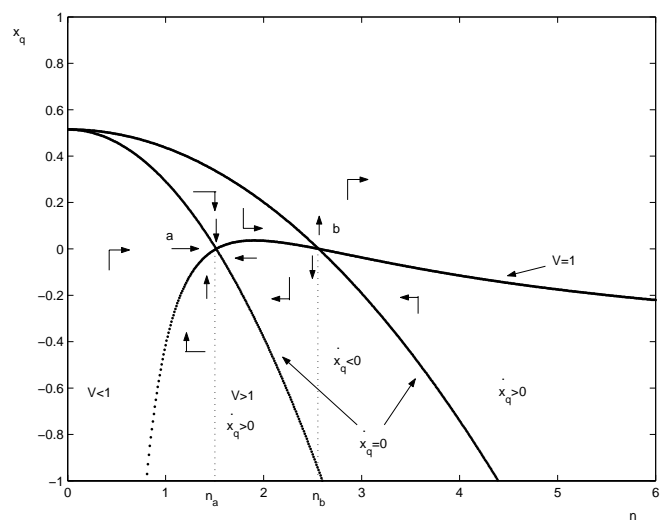

Fig. 3. Qualitative picture of (4) and (5).

given by,

$$
\begin{aligned}
& \dot{x}_{q}=0: \\
& \quad x_{q}=Q_{s}-\frac{Q_{s} n^{2}}{2}\left(V_{0}^{2}-2 x Q_{s} \pm V_{0} \sqrt{V_{0}^{2}-4 x Q_{s}}\right) \\
& V=1: \quad x_{q}=\frac{n V_{0}-1}{n^{2} x}-Q_{s}
\end{aligned}
$$

Figure 3 depicts these curves.

We will assume the following numerical data throughout the paper: $Q_{s}=0.515 \mathrm{pu}, V_{0}=1.05 \mathrm{pu}, x=0.5 \mathrm{pu}, T_{q}=60 \mathrm{sec}$, $T=30 \mathrm{sec}$. Also, it is assumed that the transformer AVR deadband has negligible width, i.e., $V_{L}=V_{H}=V_{m}^{0}$.

It can be seen from Figure 3 that the $\dot{x}_{q}=0$ and $V=1$ curves partition the state space into distinct regions where $\dot{x}_{q}<0$ or $\dot{x}_{q}>0$, and $V<1$ or $V>1$ (and their possible combinations). The curves intersect at two distinct points $a$ and $b$. It follows that trajectories in the neighborhood of $a$ move towards $a$, but will not reach $a$ if $n_{a} \notin \mathbf{Q}$. Hence, there exists an invariant region around $a$, which may be a stable limit cycle. Trajectories around $b$ do not behave in this manner. In fact, they move away from $b$, ruling out the existence of a stable limit cycle around $b$. Indeed, the section of the curve $\dot{x}_{q}=0$ that includes point $b$ is a locus of unstable equilibria.

The focus is therefore on trajectories around $a$. A Poincaré map will be used to prove the existence of a locally stable limit cycle.

\section{POINCARÉ MAPS}

Poincaré map concepts can be used to determine the local stability of limit cycles. To summarize this approach, let there be a hyperplane transversal to the limit cycle at an arbitrary point on it. Trajectories originating from that hyperplane in a neighborhood of the limit cycle encounter the hyperplane again after about $t_{p}$ seconds, where $t_{p}$ is the period of the limit cycle. Thus, a Poincare map samples the flow of a periodic system once every period. This sampling process can be written

$$
x^{k+1}=P\left(x^{k}\right)
$$

where $P$ denotes the Poincaré map and $x^{k}$ is the $k^{t h}$ sample point of the flow. Differentiating (6) gives the linearized Poincaré map

$$
\Delta x^{k+1}=D P \Delta x^{k}
$$

where $D$ is the differential operator. Small perturbations in $x^{k}$ diminish if the eigenvalues of $D P$ are all less than 1 . In that case the limit cycle is locally stable, as any local trajectory is attracted to the limit cycle. Full details can be found in $[5,6]$.

\section{LOCAL STABILITY OF TAP-CHANGER INDUCED LIMIT CYCLE}

If the equilibrium value of tap position $n_{a} \notin \mathbf{Q}$, the system will not be able to stabilize to an equilibrium point. Instead it will continually switch between tap positions $n_{1}, n_{2} \in \mathbf{Q}$, where $n_{1}<$ $n_{a}<n_{2}$. This section assumes the existence of limit cycle behavior, and uses a Poincaré map to prove local stability. Conditions governing the existence of a limit cycle, along with global stability issues, are presented in the later section.

The continuous dynamic behavior of the system is described by (1)-(3). Eliminating algebraic variable $V$ results in the form,

$$
\dot{x}_{q}=f\left(x_{q} ; n\right), \quad n \in \mathbf{Q} .
$$

The full hybrid dynamics also involve switching of $n$ at every $T$ seconds, as presented in Figure 1. The complete model can be written in a differential algebraic impulsive switched (DAIS) form [6]. The flow of the system may be expressed in the implicit form

$$
\phi\left(x_{q}, x_{q}(0), t ; n_{1}, n_{2}\right)=0 .
$$

The Poincaré map samples the trajectory every $2 T$ seconds, since the switching is periodic. Let $x_{q}^{k}$ and $x_{q}^{k+1}$ be consecutive samples. The map that translates $x_{q}^{k}$ to $x_{q}^{k+1}$ is given by

$$
\phi\left(x_{q}^{k+1}, x_{q}^{k}, 2 T ; n_{1}, n_{2}\right)=0 .
$$

Progression from $x_{q}^{k}$ to $x_{q}^{k+1}$ involves the evolution of the system (8) with $n=n_{1}$ for $T$ seconds, followed by $n=n_{2}$ for a further $T$ seconds. Therefore (10) can be decomposed as

$$
\begin{aligned}
\phi^{\prime}\left(x_{q}^{k^{\prime}}, x_{q}^{k}, T ; n_{1}\right) & =0 \\
\phi^{\prime}\left(x_{q}^{k+1}, x_{q}^{k^{\prime}}, T ; n_{2}\right) & =0
\end{aligned}
$$

where $x_{q}^{k^{\prime}}$ is the point when switching from $n_{1}$ to $n_{2}$ takes place.

To obtain the linearized map, (8) can be rearranged and integrated over $T$ seconds, giving

$$
\underbrace{\int_{x_{q}^{k}}^{x_{q}^{k^{\prime}}} \frac{d x_{q}}{f\left(x_{q} ; n_{1}\right)}-T}_{\phi^{\prime}\left(x_{q}^{k^{\prime}}, x_{q}^{k}, T ; n_{1}\right)}=0
$$

Linearizing (11) gives

$$
\frac{\partial \phi^{\prime}\left(x_{q}^{k^{\prime}}, x_{q}^{k}, T ; n_{1}\right)}{\partial x_{q}^{k^{\prime}}} \Delta x_{q}^{k^{\prime}}+\frac{\partial \phi^{\prime}\left(x_{q}^{k^{\prime}}, x_{q}^{k}, T ; n_{1}\right)}{\partial x_{q}^{k}} \Delta x_{q}^{k}=0 .
$$

Therefore

$$
\begin{aligned}
& \frac{\partial}{\partial x_{q}^{k^{\prime}}}\left(\int_{x_{q}^{k}}^{x_{q}^{k^{\prime}}} \frac{d x_{q}}{f\left(x_{q} ; n_{1}\right)}-T\right) \Delta x_{q}^{k^{\prime}}+ \\
& \frac{\partial}{\partial x_{q}^{k}}\left(\int_{x_{q}^{k}}^{x_{q}^{k^{\prime}}} \frac{d x_{q}}{f\left(x_{q} ; n_{1}\right)}-T\right) \Delta x_{q}^{k}=0 \\
\Rightarrow \quad & \frac{1}{f\left(x_{q}^{k^{\prime}} ; n_{1}\right)} \Delta x_{q}^{k^{\prime}}-\frac{1}{f\left(x_{q}^{k} ; n_{1}\right)} \Delta x_{q}^{k}=0 .
\end{aligned}
$$


Hence

Similarly,

$$
\Delta x_{q}^{k^{\prime}}=\frac{f\left(x_{q}^{k^{\prime}} ; n_{1}\right)}{f\left(x_{q}^{k} ; n_{1}\right)} \Delta x_{q}^{k}
$$

$$
\Delta x_{q}^{k+1}=\frac{f\left(x_{q}^{k+1} ; n_{2}\right)}{f\left(x_{q}^{k^{\prime}} ; n_{2}\right)} \Delta x_{q}^{k^{\prime}} .
$$

The complete linearized mapping is given by

$$
\Delta x_{q}^{k+1}=\underbrace{\frac{f\left(x_{q}^{k+1} ; n_{2}\right)}{f\left(x_{q}^{k^{\prime}} ; n_{2}\right)} \cdot \frac{f\left(x_{q}^{k^{\prime}} ; n_{1}\right)}{f\left(x_{q}^{k} ; n_{1}\right)}}_{D P\left(x_{q}^{k+1}, x_{q}^{k^{\prime}}, x_{q}^{k}, 2 T ; n_{1}, n_{2}\right)} \Delta x_{q}^{k} .
$$

The value of $D P$ can be explicitly calculated for the system under consideration, as $x_{q}^{k^{\prime}}$ and $x_{q}^{k+1}$ are easily computed by numerical iterations, for given $x_{q}^{k}$. For $n_{1}, n_{2}$ and $x_{q}^{k}$ in a small neighborhood around $a$ with $n_{1}<n_{a}<n_{2}, D P<1$. For example, $n_{1}=1.4, n_{2}=1.6, x_{q}^{k}=0$ gives $D P=0.764<1$. Thus, the limit cycle around $a$ is locally stable. Near point $b, n_{1}=2.5$, $n_{2}=2.7$ and $x_{q}^{k}=0$, resulting in a value of $D P=1.32>1$. Thus no stable limit cycle exists around $b$. This supports the earlier conjecture.

Similar analysis is applicable to higher dimensional hybrid system. In general though $D P$ cannot be explicit formed, but is obtained from trajectory sensitivities [6]. The eigenvalues of $D P$ i.e. characteristic multipliers, determine the local stability of the hybrid limit cycle [5].

\section{GLOBAL STABILITY OF HYBRID LIMIT CYCLE}

Linearization of the Poincaré map does not allow investigation of the region of attraction of the limit cycle. Unfortunately the implicit form of (10) complicates such analysis. However by employing a reasonable approximation, $x_{q}^{k+1}$ can be expressed explicitly in terms of $x^{k}$, facilitating the desired analysis.

Equations (2)-(3) can be manipulated to give

$$
V^{2}=\frac{\left(\frac{V_{0}^{2}}{n x}\right)^{2}-2 x_{q}\left(Q_{s}+\frac{1}{n^{2} x}\right) \pm\left(\frac{V_{0}^{2}}{n x}\right)^{2} \sqrt{1-\frac{4 x_{q}\left(Q_{s}+\frac{1}{n^{2} x}\right)}{\left(\frac{V_{0}^{2}}{n x}\right)^{2}}}}{2\left(Q_{s}+\frac{1}{n^{2} x}\right)^{2}} .
$$

Using an approximation $\sqrt{1+z} \approx 1+\frac{z}{2}$, for $|z| \ll 1$, we have for $\left|\frac{4 x_{q}\left(Q_{s}+\frac{1}{n^{2} x}\right)}{\left(\frac{V_{0}}{n x}\right)^{2}}\right| \ll 1$, (ignoring the trivial solution $V^{2}=0$ ),

$$
V^{2} \approx \frac{\left(\frac{V_{0}}{n x}\right)^{2}-2 x_{q}\left(Q_{s}+\frac{1}{n^{2} x}\right)}{\left(Q_{s}+\frac{1}{n^{2} x}\right)^{2}} .
$$

Using (17), the system (1)-(3) can now be described in terms of $x_{q}$ and $n$ as

$$
\dot{x}_{q}=a(n) x_{q}+b(n)
$$

where

$$
\begin{aligned}
& a(n)=\frac{1}{T_{q}}\left(\frac{Q_{s}-\frac{1}{n^{2} x}}{Q_{s}+\frac{1}{n^{2} x}}\right) \\
& b(n)=\frac{Q_{s}}{T_{q}}\left(1-\left(\frac{\frac{V_{0}}{n x}}{Q_{s}+\frac{1}{n^{2} x}}\right)^{2}\right) .
\end{aligned}
$$

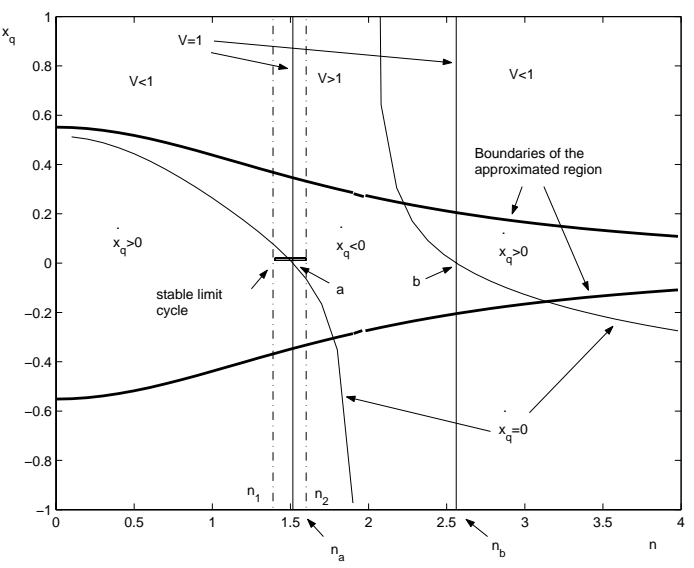

Fig. 4. Boundaries of the approximated region and a qualitative picture of (19) and (20).

Using the parameter values for the example system, the approximation is valid for $x_{q}^{2} \ll \frac{1.2155}{\left(2+0.515 n^{2}\right)^{2}}$. Figure 4 shows the curves $x_{q}^{2}=\frac{1.2155}{\left(2+0.515 n^{2}\right)^{2}}$, which serve as boundaries for the region of approximation. As the limit cycle is well within that region, the approximated system can be used to derive conditions for limit cycle stability.

For the approximate system, the curves $V=1$ and $\dot{x}_{q}=0$ are given by,

$$
\begin{aligned}
& V=1: \frac{V_{0}}{n x}=Q_{s}+\frac{1}{n^{2} x} \\
& \quad \Rightarrow n=n_{a}=1.516 \text { and } n=n_{b}=2.5616 \\
& \dot{x}_{q}=0: x_{q}=-\frac{b(n)}{a(n)}=\frac{Q_{s}\left(\left(\frac{V_{0}}{n x}\right)^{2}-\left(Q_{s}+\frac{1}{n^{2} x}\right)^{2}\right)}{\left(Q_{s}+\frac{1}{n^{2} x}\right)\left(Q_{s}-\frac{1}{n^{2} x}\right)}
\end{aligned}
$$

Equation (19) is obtained by setting $V=1$ in (17) and reusing the approximation $\left|\frac{4 x_{q}\left(Q_{s}+\frac{1}{n^{2} x}\right)}{\left(\frac{V_{0}}{n x}\right)^{2}}\right| \ll 1$. These curves are plotted in Figure 4. (Compare with Figure 3.) The intersection points are the same as before, but the curves are qualitatively different.

Consider now a system described by (18) which is an approximated version of (1)-(3). For a fixed value of $n$, the load state $x_{q}(t)$ is given by

$$
x_{q}(t)=-\frac{b(n)}{a(n)}+\left(x_{q}(0)+\frac{b(n)}{a(n)}\right) e^{a(n) t}
$$

Let the tap positions be restricted to $\mathbf{Q}=\left\{n_{1}, n_{2}: n_{1}<n_{2}\right\}$. A hybrid system is obtained by switching between $n=n_{1}$ and $n=n_{2}$ every $T$ seconds. Conditions are sought on $n_{1}, n_{2}$ and $x_{q}(0)$ which ensure the existence of a limit cycle that is attracting over the approximated region. A map from $x_{q}^{k}$ to $x_{q}^{k+1}$ can be obtained using (11)-(12) and (21),

$$
\begin{aligned}
x_{q}^{k^{\prime}} & =-\frac{b\left(n_{1}\right)}{a\left(n_{1}\right)}+\left(x_{q}^{k}+\frac{b\left(n_{1}\right)}{a\left(n_{1}\right)}\right) e^{a\left(n_{1}\right) T} \\
x_{q}^{k+1} & =-\frac{b\left(n_{2}\right)}{a\left(n_{2}\right)}+\left(x_{q}^{k^{\prime}}+\frac{b\left(n_{2}\right)}{a\left(n_{2}\right)}\right) e^{a\left(n_{2}\right) T} .
\end{aligned}
$$


The complete mapping is

$$
\begin{aligned}
x_{q}^{k+1}= & -\frac{b\left(n_{2}\right)}{a\left(n_{2}\right)}-\left(\frac{b\left(n_{1}\right)}{a\left(n_{1}\right)}-\frac{b\left(n_{2}\right)}{a\left(n_{2}\right)}\right) e^{a\left(n_{2}\right) T} \\
& +\left(x_{q}^{k}+\frac{b\left(n_{1}\right)}{a\left(n_{1}\right)}\right) e^{\left(a\left(n_{1}\right)+a\left(n_{2}\right)\right) T} .
\end{aligned}
$$

The stationary point of the mapping, if it exists, is obtained by setting $x_{q}^{k+1}=x_{q}^{k}=x_{q}^{*}$ and is given by

$$
x_{q}^{*}=\frac{\frac{b\left(n_{2}\right)}{a\left(n_{2}\right)}+\left[\frac{b\left(n_{1}\right)}{a\left(n_{1}\right)}-\frac{b\left(n_{2}\right)}{a\left(n_{2}\right)}\right] e^{a\left(n_{2}\right) T}-\frac{b\left(n_{1}\right)}{a\left(n_{1}\right)} e^{\left[a\left(n_{1}\right)+a\left(n_{2}\right)\right] T}}{e^{\left[a\left(n_{1}\right)+a\left(n_{2}\right)\right] T}-1} .
$$

Consider the sequence

$$
\left\{x_{q}^{0}, x_{q}^{1}, x_{q}^{2}, \ldots, x_{q}^{k}, \ldots\right\}
$$

In general, $x_{q}^{k}$ may be expressed, after some manipulations, as

$$
x_{q}^{k}=x_{q}^{*}+\left(x_{q}^{0}-x_{q}^{*}\right) e^{k\left(a\left(n_{1}\right)+a\left(n_{2}\right)\right) T}
$$

As $x_{q}^{0} \neq x_{q}^{*}$ in general, $x_{q}^{k} \rightarrow x_{q}^{*}$ as $k \rightarrow \infty$ iff $a\left(n_{1}\right)+a\left(n_{2}\right)<$ 0 . Hence, a necessary condition for a stable mapping is

$$
a\left(n_{1}\right)+a\left(n_{2}\right)<0,
$$

that is, the average system must be stable. Further, it is evident that a Lyapunov function $\mathcal{V}=\left(x_{q}-x_{q}^{*}\right)^{2}$ defined on the switching surface decreases after every $2 T$ seconds if $a\left(n_{1}\right)+a\left(n_{2}\right)<0$.

A stable mapping implies the existence of a stationary point $x_{q}^{*}$, and rules out the possibility of chaotic behavior of the hybrid system. But it does not necessarily imply the existence of a limit cycle, as $x_{q}^{*}$ could be an equilibrium point. However consider the sequence

$$
\left\{x_{q}^{0^{\prime}}, x_{q}^{1^{\prime}}, x_{q}^{2^{\prime}}, \ldots, x_{q}^{k^{\prime}}, \ldots\right\}
$$

The sequences (24) and (27) converge to $x_{q}^{*}$ and $x_{q}^{* \prime}$ respectively, subject to $a\left(n_{1}\right)+a\left(n_{2}\right)<0$, where

$x_{q}^{* \prime}=\frac{\frac{b\left(n_{1}\right)}{a\left(n_{1}\right)}+\left[\frac{b\left(n_{2}\right)}{a\left(n_{2}\right)}-\frac{b\left(n_{1}\right)}{a\left(n_{1}\right)}\right] e^{a\left(n_{1}\right) T}-\frac{b\left(n_{2}\right)}{a\left(n_{2}\right)} e^{\left[a\left(n_{1}\right)+a\left(n_{2}\right)\right] T}}{e^{\left[a\left(n_{1}\right)+a\left(n_{2}\right)\right] T}-1}$.

If $x_{q}^{*}=x_{q}^{* \prime}$, then the sequence

$$
\left\{x_{q}^{0}, x_{q}^{0^{\prime}}, x_{q}^{1}, x_{q}^{1^{\prime}}, \ldots, x_{q}^{k}, x_{q}^{k^{\prime}}, \ldots\right\}
$$

converges and $x_{q}^{*}$ is an equilibrium point. If not, it eventually oscillates between $x_{q}^{*}$ and $x_{q}^{*^{\prime}}$ giving rise to a limit cycle. Thus, limit cycle existence requires $x_{q}^{*} \neq x_{q}^{* \prime}$, which is equivalent to (18) having a different equilibrium point for $n_{1}$ versus $n_{2}$, i.e.,

$$
-b\left(n_{1}\right) / a\left(n_{1}\right) \neq-b\left(n_{2}\right) / a\left(n_{2}\right) .
$$

As any convergent sequence of real numbers converges to a unique limit, (26) and (29) ensure a unique limit cycle for fixed $n_{1}$ and $n_{2}$.

The tap changing strategy described in Section 2 requires that a tap change from $n_{1}$ to $n_{2}\left(n_{1}<n_{2}\right)$ take place when $V\left(x_{q}(\tau), n_{1}\right)<1$ and from $n_{2}$ to $n_{1}$ when $V\left(x_{q}(\tau), n_{2}\right)>1$, where $\tau$ is the switching time instant. For the approximate system (18), and using (19), these conditions are translated into,

$$
\frac{V_{0}}{n_{1} x}>Q_{s}+\frac{1}{n_{1}^{2} x}, \quad \frac{V_{0}}{n_{2} x}<Q_{s}+\frac{1}{n_{2}^{2} x} .
$$

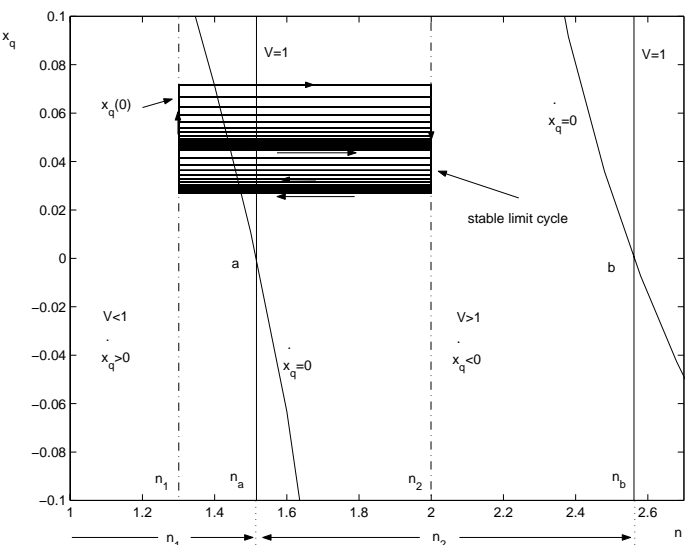

Fig. 5. Conditions on $n_{1}$ and $n_{2}$ for a stable limit cycle.

Conditions (26), (30), along with $x_{q}^{*} \neq x_{q}^{* \prime}$, when grouped together simply imply $0<n_{1}<n_{a}$ and $n_{a}<n_{2}<n_{b}$. If $x_{q}(0)$ is well within the region of approximation, this ensures the existence of a stable limit cycle for the system (18) with $\mathbf{Q}=\left\{n_{1}, n_{2}\right.$ : $\left.n_{1}<n_{2}\right\}$. Figure 5 illustrates convergence to a stable limit cycle when the above restrictions on $n_{1}$ and $n_{2}$ are imposed.

\section{CONCLUSIONS}

A relatively simple hybrid system, consisting of a tap changing transformer and a dynamic load, has been analysed. Conditions which ensure a unique stable limit cycle were determined. This analysis relied on a reasonable approximation which resulted in an explicit expression for a Poincaré map. Stability analysis of more general load-transformer systems is difficult. Nevertheless, the ideas and intuition from this example form the basis for analysing more realistic and complex hybrid systems. This is the focus of on-going research.

\section{REFERENCES}

[1] M. Larsson, D.H. Popović, and D.J. Hill, "Limit cycles in power systems due to OLTC deadbands and load-voltage dynamics," Electric Power Systems Research, vol. 47, pp. 181$188,1998$.

[2] D.J. Hill, "Nonlinear dynamic load models with recovery for voltage stability studies," IEEE Transactions on Power Systems, vol. 8, no. 1, pp. 166-176, February 1993.

[3] D.H. Popović, I.A. Hiskens, and D.J. Hill, "Investigations of load-tap changer interactions," International Journal of Electric Power and Energy Systems, vol. 18, no. 2, pp. 81-97, 1996.

[4] K.T. Vu and C-C. Liu, "Shrinking stability regions and voltage collapse in power systems," IEEE Transactions on Circuits and Systems I, vol. 39, no. 4, pp. 271-289, April 1992.

[5] T.S Parker and L.O. Chua, Practical Numerical Algorithms for Chaotic Systems, Springer-Verlag, New York, NY, 1989.

[6] I.A. Hiskens, "Stability of hybrid system limit cycles: Application to the compass gait biped robot," in Proceedings of the 40th IEEE Conference on Decision and Control, Orlando, FL, December 2001. 